\title{
Directionality of $o$-Phthalaldehyde adsorbed onto H-passivated Si(100) Surface Characterized by NEXAFS and HRPES
}

\author{
Ki-jeong Kim, Sena Yang, ${ }^{\dagger}$ Tai-Hee Kang, Bongsoo Kim, ${ }^{*}$ and Hangil Lee ${ }^{\dagger, *}$ \\ Pohang Accelerator Laboratory, POSTECH, Pohang 790-784, Korea. *E-mail: kbs007@postech.ac.kr \\ ${ }^{\dagger}$ Department of Chemistry, Sookmyung Women's University, Seoul 140-742, Korea. *E-mail: easyscan@sookmyung.ac.kr \\ Received April 28, 2010, Accepted May 22, 2010
}

\begin{abstract}
The electronic and adsorption structure of $o$-phthalaldehyde (OPA) on the H-Si(100) surface was investigated by using Near Edge X-ray Fine Structure (NEXAFS) and high resolution photoemission spectroscopy (HRPES). We confirmed that the OPA grown on the $\mathrm{H}-\mathrm{Si}(100)$ surface showed good dependency with about 60 degree tilting angle using NEXAFS and a single $\mathrm{O} 1 \mathrm{~s}$ peak by using HRPES. Hydrogen atom passivated on the $\mathrm{Si}(100)$ surface was found to be a seed for making one dimensional organic line that uses a chain reaction as the $\mathrm{H}-\mathrm{Si}(100)$ surface was compared with the hydrogen free $\operatorname{Si}(100)$ surface.
\end{abstract}

Key Words: $o$-Phthalaldehyde, H-Si(100), HRPES, NEXAFS, 1-D Organic molecular line

\section{Introduction}

For several decades, the fabrication of organic nanostructures and their self-assembling control on silicon surfaces has been intensively studied because of their versatile potential application as a silicon-based electronic components as well as the connecting wire in a variety of molecular scale devices. ${ }^{1-6}$ Especially, the manipulation of one-dimensional (1D) organic nanostructures on the hydrogen terminated $\mathrm{Si}(100)(\mathrm{H}-\mathrm{Si}(100))$ surface has been researched not only for industrial applications but also for academic interest. ${ }^{4,7}$

Generally, to make one-dimensional (1D) organic nanostructures, the radical species must succeed in abstracting a hydrogen atom from an adjacent $\mathrm{Si}-\mathrm{H}$ surface site through chain reactions. As a result, it must generate a stable chemisorption between the organic molecule and the consecutive chain reactions using radical included in hydrogen atom. ${ }^{8}$ The organic layers and lines on silicon surfaces have been a very interesting research subject from both scientific and technological points of view. The hybrid between the organic materials and the chain reactions is of particular interest since 1D line growth might provide the connecting wire in molecular devices and in the patterning of molecular nano scale devices. A density functional calculation revealed that $o$-phthaladehyde (OPA) grows 1D molecular lines self-directly on the H-terminated $\mathrm{Si}(001)$ surface.

Recently, Cho et al. have reported an interesting theoretical results that $\mathrm{OPA}\left(\mathrm{C}_{8} \mathrm{H}_{6} \mathrm{O}_{2}\right)$ molecule on $\mathrm{H}-\mathrm{Si}(100)$ can be formed into $1 \mathrm{D}$ organic molecular line using a chain reaction ${ }^{9}$ and the mechanism of it. ${ }^{10}$ To clarify the formation of $1 \mathrm{D}$ organic molecular line that uses OPA, we performed a near edge x-ray adsorption fine structure (NEXAFS) and high resolution photoemission spectroscopy (HRPES) experiment using synchrotron radiation.

\section{Experimentals}

An n-type $\operatorname{Si}(100)$ sample with a resistivity of $0.1 \Omega \mathrm{cm}$ was cleaned by repetitive heating to $1200{ }^{\circ} \mathrm{C}$ after outgassing at
$600{ }^{\circ} \mathrm{C}$ for 12 hours; the cleanliness of the $\mathrm{Si}(100)-2 \times 1$ surface was confirmed by HRPES. We used hydrogen plasma dozer to prepare H-terminated $\mathrm{Si}(100)$ surface. We maintained the temperature of $\mathrm{Si}(100)-2 \times 1$ to room temperature and back filled the hydrogen gas to the base pressure was $5 \times 10^{-8}$ Torr during $2 \mathrm{~min}$ at the filament temperature of $1580{ }^{\circ} \mathrm{C}$. And then we confirmed the formation of $\mathrm{H}-\mathrm{Si}$ bond through the $\mathrm{Si} 2 p$ core level spectra. OPA $\left(\mathrm{C}_{8} \mathrm{H}_{6} \mathrm{O}_{2}\right.$ : see Figure 1) was purchased from SIGMA ALDRICH ( $>99.0 \%$ purity) and further purified through several freeze-pump-thaw cycles to remove all dissolved gases before being exposed to clean $\mathrm{Si}(100)-2 \times 1$. The purity of OPA was verified using in situ mass spectrometry in a vacuum chamber. OPA was exposed onto $\mathrm{Si}(100)-2 \times 1$ at room temperature through a direct doser controlled by a variable leak valve. The pressure during OPA exposure was determined from the uncorrected ion gauge reading. The exposure unit is Langmuir (L) $\left(1 \mathrm{~L}=1 \times 10^{-6}\right.$ Torr $\left.\cdot \mathrm{sec}\right)$.

$\mathrm{C} 1 s, \mathrm{O} 1 s$ and $\mathrm{Si} 2 p$ core level spectra were measured using the photon energy of $330,635,135 \mathrm{eV}$ at $8 \mathrm{~A} 2 \mathrm{HR}$-PES beamline in the Pohang accelerator laboratory (PAL). The binding energy and the spectral resolution of $\mathrm{C} 1 s, \mathrm{O} 1 s$ and $\mathrm{Si} 2 p$ were calibrated by measuring the $\mathrm{Au} 4 f_{7 / 2}$ core level and the $\mathrm{Au}$ Fermi edge. The base pressure of the chamber was maintained below $2.0 \times 10^{-10}$ torr. All the spectra were recorded in the normal emission mode. The photoemission spectra were carefully analyzed by using a standard nonlinear least squares fitting

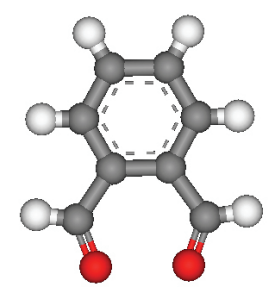

Figure 1. The structure of $o$-phthaladehyde molecule. Each gray, white, red colored ball indicates carbon $(\mathrm{C})$, hydrogen $(\mathrm{H})$, and oxygen $(\mathrm{O})$, respectively. 


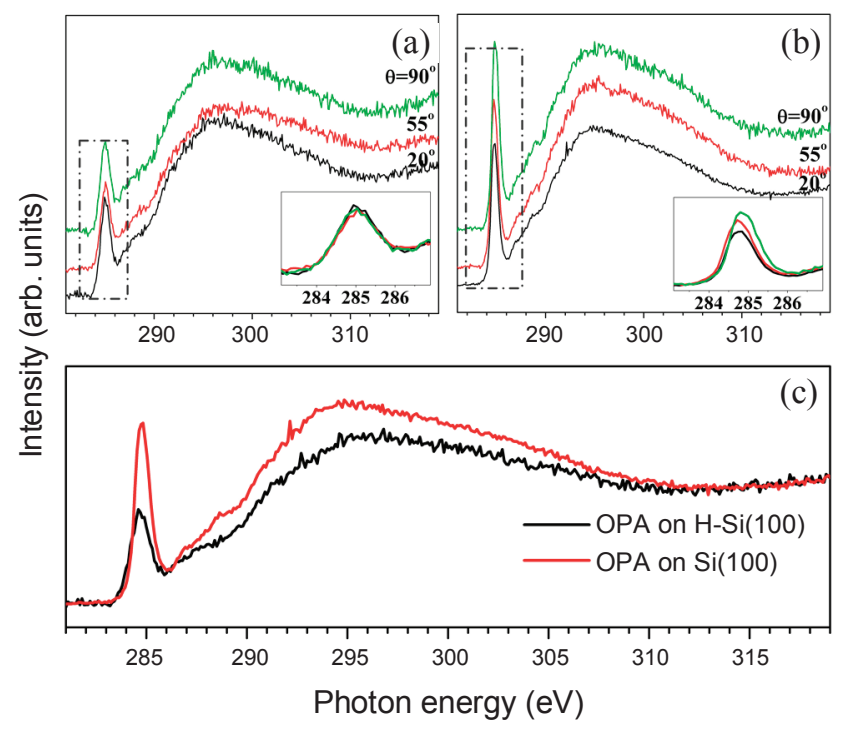

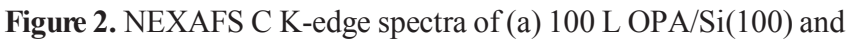
(b) $100 \mathrm{~L} \mathrm{OPA} / \mathrm{H}-\mathrm{Si}(100)$ surface. (c) Comparison of the intensities of samples prepared under different conditions.

procedure with Voigt functions. ${ }^{11}$

The NEXAFS spectra were measured at 2B1 PES beamline in the partial electron yield (PEY) detection mode with a retarding voltage of $-210 \mathrm{~V}$ and an accelerating voltage of $1.6 \mathrm{kV} .^{12}$ Molecular bonding information for the top few layers of the multilayer films was obtained from the PEY mode NEXAFS spectra by considering probing depths of less than $10 \AA .{ }^{13}$ The photons had a degree of polarization of about $85 \%$, with an incident photon energy resolution of $350 \mathrm{meV}$ near the carbon K-edge region.

\section{Data and Results}

First, C K-edge NEXAFS spectra were acquired. Figure 2(a) displays $\mathrm{C}$ K-edge spectra for the OPA adsorbed onto the $\mathrm{Si}(100)$ surface, and Figure 2(b) shows the C K-edge spectra for the OPA adsorbed onto the hydrogen-passivated $\mathrm{Si}(100)$ surfaces using a different incidence angle.

An OPA molecule adsorbed onto the hydrogen-free $\mathrm{Si}(100)$ surface, as shown in Figure 2(a), was distinctly different in structure from an OPA molecule adsorbed onto the $\mathrm{H}-\mathrm{Si}(100)$ surface. The intensity of the $\mathrm{C}=\mathrm{C} \pi^{*}$ was found to be independent of the incident angle $\theta$. In contrast, OPA molecules adsorbed onto the $\mathrm{H}-\mathrm{Si}(100)$ surface, shown in Figure 2(b), showed an angular dependence of the $\mathrm{C}=\mathrm{C} \pi *$ intensity at a photon energy of $285 \mathrm{eV}$. The insets of Figures 2(a) and 2(b) show the intensity variations as a function of photon incident angle $\theta$. The inset of Figure 2(b) in particular shows good angular dependence, indicating that the OPA molecules were uniformly oriented on the $\mathrm{H}-\mathrm{Si}(100)$ surface. Figure 2(c) compares the intensity variations of $\mathrm{C}=\mathrm{C} \pi^{*}$ at $\theta=20^{\circ}$. As shown, the intensity obtained from OPA on $\mathrm{H}-\mathrm{Si}(100)$ decreased to almost $52.2 \%$ of the intensity observed from the OPA adsorbed onto the hydrogen-free $\mathrm{Si}(100)$ surface. Due to the presence of the reactive $\mathrm{Si}=\mathrm{Si}$ dimer on the $\mathrm{Si}(100)-2 \times 1$ surface, OPA adsorbed with

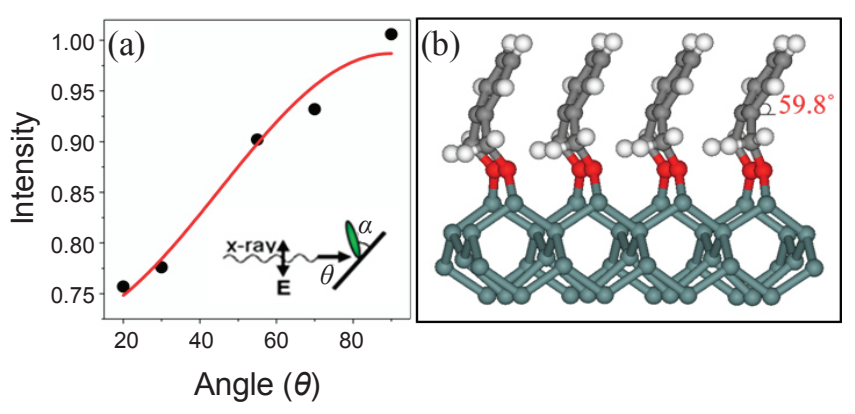

Figure 3. (a) Intensity of the $\pi^{*}(\mathrm{C}=\mathrm{C})$ resonance of OPA on $\mathrm{H}-\mathrm{Si}(100)$ $2 \times 1$ as a function of the incidence angle. (b) Schematic diagram of the OPA adsorbed onto the H-Si(100) surface. Gray, white, red, and dark cyan colored balls correspond, respectively, to carbon (C), hydrogen $(\mathrm{H})$, oxygen $(\mathrm{O})$, and the $\mathrm{Si}(100)$ surface.

random orientations onto the $\mathrm{Si}(100)-2 \times 1$ surface.

The OPA $\mathrm{C}=\mathrm{C} \pi *$ intensity on the $\mathrm{H}-\mathrm{Si}(100)$ surface clearly showed an angular dependence (Figure 2(b)). The average tilt angle between the double bonds of the OPA and the $\mathrm{Si}(100)-2 \times 1$ substrate was determined using an analytical solution to the NEXAFS intensity problem. The relationship between the photoelectron intensity and the incident photon beam angle can be expressed by the equation. ${ }^{13,14}$

$$
I_{v}=C\left[\frac{P}{3}\left\{1+\frac{1}{2}\left(3 \cos ^{2} \theta-1\right)\left(3 \cos ^{2} \alpha-1\right)\right\}+\frac{(1-P)}{2} \sin ^{2} \alpha\right]
$$

where $\alpha$ and $\theta$ are the angle between the molecular axis and the surface normal and the angle between the light polarization vector (E-vector) and the surface normal, respectively, and $P$ is the degree of polarization.

For this experiment, we assumed a polarization factor of $P=$ 0.85 . Moreover, the tilting angle of the OPA molecules adsorbed onto the $\mathrm{H}-\mathrm{Si}(100)$ surface was calculated using the experimentally measured relative intensity of the $285.0 \mathrm{eV} \pi^{*}(\mathrm{C}=\mathrm{C})$ resonance as a function of the polarization vector angle.

Figure 3(a) shows the intensity of the $\pi^{*}(\mathrm{C}=\mathrm{C})$ resonance of OPA on $\mathrm{H}-\mathrm{Si}(100)-2 \times 1$ surfaces as a function of the incidence angle. Quantitative analysis revealed an average tilt angle of $59.8^{\circ}$. Figure $3(\mathrm{~b})$ illustrates the adsorption geometry obtained from the two distinct spectra.

HRPES spectra were acquired to characterize the mechanism of 1D molecular growth on the H-terminated $\mathrm{Si}(100)$ surface. A single or double oxygen peak in the HRPES spectrum would be characteristic of an autocatalytic chain reaction. The left panel shows the Si $2 p$ core-level spectra for samples of 1D organic nanostructures prepared by a variety of methods. The $\mathrm{Si}$ $2 p$ peak of the clean $\mathrm{Si}(100)-2^{\prime} 1$ surface was decomposed into four peaks that reflected the surface states of the dangling bond $\left(\mathrm{S}_{\mathrm{u}}\right)$, the second layer $(\mathrm{S})$, components originating from defects (D), and bulk (B). After confirming the clean Si $2 p$ core-level spectrum (see Figure 4(a)), atomic hydrogen was deposited using a plasma hydrogen doser to terminate the $\mathrm{Si}(100)$ surface. As shown in Figure 4(b), the peak corresponding to $\mathrm{Si}-\mathrm{H}$ appeared at a higher binding energy $(100.1 \mathrm{eV})$, and the surface peak (marked as $\mathrm{S}_{\mathrm{u}}$ ) disappeared completely, indicating that the 


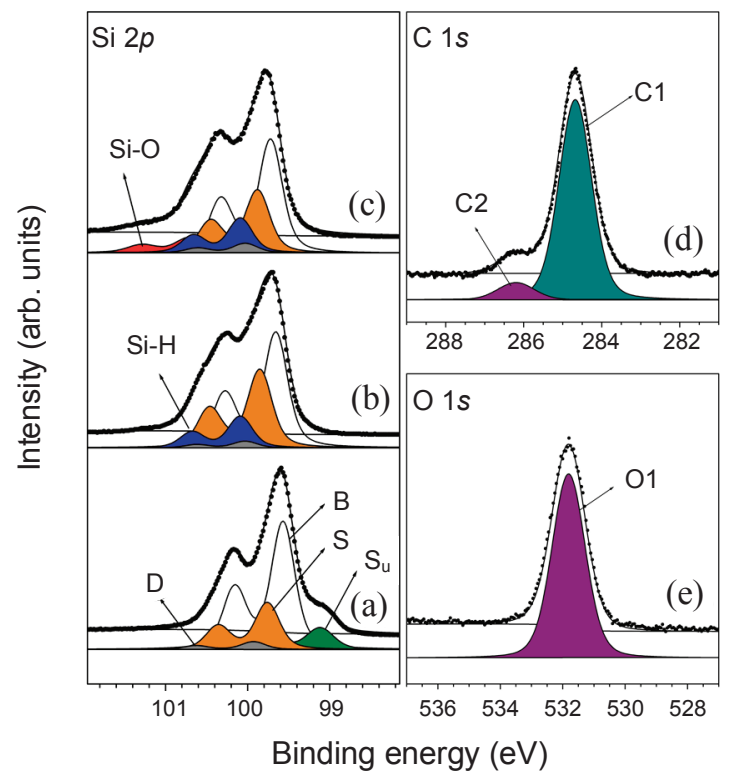

Figure 4. High resolution photoemission spectra. Left panel: Si $2 p$ core level spectra (a) clean, (b) $100 \mathrm{~L}$ of hydrogen dosing, (c) $100 \mathrm{~L}$ OPA deposition on $\mathrm{H}-\mathrm{Si}(100)$ surface. Right panel: (d) C 1s and (e) $\mathrm{O} 1 \mathrm{~s}$ core-level spectrum obtained after OPA on $\mathrm{H}-\mathrm{Si}(100)$ surface.

$\mathrm{Si}(100)$ surface was completely covered with hydrogen. Finally, the OPA was deposited onto the H-terminated $\mathrm{Si}(100)$ surface to study the autocatalytic chain reaction. Figure 4(c) shows the Si $2 p$ core-level spectrum obtained after OPA deposition on the $\mathrm{H}-\mathrm{Si}(100)$ surface. The Si-O related peak was observed at a binding energy of $100.7 \mathrm{eV}$.

The focus of this study was the growth of 1D organic molecular nanostructures aligned along a dimer row. Therefore, the $\mathrm{C} 1 s$ and $\mathrm{O} 1 s$ peaks of OPA also required examination. Figure 4(d) shows the C $1 s$ core-level spectrum obtained after OPA deposition. As expected, two distinct carbon peaks were observed: one peak corresponded to the phenyl ring at $284.7 \mathrm{eV}$ and the other peak originated from the carbonyl group at $286.1 \mathrm{eV}$. Figure 4(e) shows the O $1 s$ core-level spectrum. As mentioned, the oxygen peak was the most important feature required for confirmation of the OPA chain reaction. As shown in this figure, a single oxygen peak at $531.8 \mathrm{eV}$ indicated the chain reaction, as expected. These findings suggested that OPA growth on the H-Si(100) surface was accompanied by the simultaneous formation of Si-O due to radical transformation.

\section{Conclusion}

We investigated the growth and structure of a 1D organic molecular nanostructure on a H-terminated $\mathrm{Si}(100)$ surface via OPA chain reaction using NEXAFS and HRPES characterization methods. Two important experimental results were obtained: 1) NEXAFS showed that the OPA molecule grew on the $\mathrm{H}-\mathrm{Si}(100)$ surface with a $59.8^{\circ}$ molecular tilting angle, and 2) the structure was immobilized via an autocatalytic chain reaction, as confirmed by the single oxygen peak observed in HRPES.

Acknowledgments. This research was supported by the National Research Foundation of Korea funded by the Ministry of Education, Science and Technology (2010-0008608, 20090087138, and KRF-2008-314-C00169). The experiments at PLS were supported in part by MEST and POSTECH.

\section{References}

1. Collier, C. P. et al. Science 1999, 285, 391.

2. Tans, S. J.; Verschueren, A. R. M.; Dekker, C. Nature 1998, 393, 49.

3. Wolkow, R. A. Annu. Rev. Phys. Chem. 1999, 50, 413.

4. Lopinski, G. P.; Moffatt, D. J.; Wayner, D. D. M.; Walkow, R. A. Nature 1995, 392, 909.

5. Bent, S. F. Surf. Sci. 2002, 500, 879.

6. Hamers, R. J. Nature 2001, 412, 489.

7. Hossain, Md. Z.; Kato, H. S.; Kawai, M. J. Am. Chem. Soc. 2005, 127,15030 .

8. Linford, M. R.; Fenter, P.; Eisenberger, P. M.; Chidsey, C. E. D. J. Am. Chem. Soc. 1995, 117, 3145.

9. Choi, J. H.; Cho, J. H. Phys. Rev. Lett. 2007, 98, 246101.

10. Choi, J. H.; Cho, J. H. Phys. Rev. Lett. 2009, 102, 166102.

11. Schreier, F. J. Quant. Spectros. Radiat. Transfer 1992, 48, 743.

12. Lee, H.-K.; Han, J.-H.; Kim, K.-J.; Kamg, T.-H.; Kim, B. Surf. Sci. 2007, 601, 1456.

13. Stöhr, J. NEXAFS Spectroscopy; Springer: New York, 1992.

14. Stöhr, J.; Outka, D. A. Phys. Rev. B 1987, 36, 7891. 